\title{
Generation of Some Catastrophes in Optics by Binary Screens
}

\author{
Abdelmajid Belafhal*, Zoubir Hricha*, El Mostapha El Halba \\ Laboratory LPNAMME, Laser Physics Group, Department of Physics, Faculty of Sciences, Chouaïb Doukkali University, \\ El Jadida, Morocco \\ Email: hrichaz@hotmail.com, ^belafhal@gmail.com
}

How to cite this paper: Belafhal, A., Hricha, Z. and El Halba, E.M. (2019) Generation of Some Catastrophes in Optics by Binary Screens. Open Access Library Journal, 6: e5958.

https://doi.org/10.4236/oalib.1105958

Received: November 25, 2019

Accepted: December 21, 2019

Published: December 24, 2019

Copyright $\odot 2019$ by author(s) and Open Access Library Inc.

This work is licensed under the Creative Commons Attribution International License (CC BY 4.0).

http://creativecommons.org/licenses/by/4.0/

(c) (7) Open Access

\begin{abstract}
In this paper, the artificial generation of elementary catastrophe optics having odd codimensions $K=1,3$ and 5 such as the Fold, the Swallowtail and the Wigwam diffraction caustics is investigated theoretically. It is shown that the integral catastrophes with odd polynomials phase functions can be reduced to the well-known Airy-Hardy cosine integrals. In this connection, the caustic functions of the Fold, Swallowtail and Wigwam caustic beams are expressed in closed-form in terms of Airy-Hardy cosine functions. An optical method based on the Fourier transform similar to that described by Lohmann et al. [Optics Comm. 109 (1994) 361-367] is proposed for the generation of the Fold, Swallowtail and Wigwam caustic beams. The displaying of the catastrophe patterns with $K=1,3$ and 5 is optically implemented in the Fourier transform device by using simple binary screens with tailored polynomials transmission.
\end{abstract}

\section{Subject Areas}

Photochemistry

\section{Keywords}

Fold Caustic, Swallowtail Caustic, Wigwam Caustic, Airy-Hardy Integrals, Fraunhofer Diffraction Pattern, Catastrophes Optics

\section{Introduction}

In recent years, a lot of researches have been devoted to the nonconventional applications of optical computing [1]-[17]. In fact, different binary patterns have been used in many optical devices to implement intriguing phase profiles and for generating the complex amplitudes of the well-known catastrophe functions. 
The application of spatial light modulators has permitted the experimental realization of some artificial catastrophes in optics, e.g., the fundamental catastrophe beams such as the Airy (Fold) and Pearcey caustic modes [5] [16] [17]. It is well established in the catastrophe theory [18] [19] [20] [21] [22] that the catastrophe functions can be described by seven elementary structures which are classified according to their codimensions $K$. The caustic beams emerging as diffraction catastrophes are particular solutions of the paraxial Helmholtz equation, and they can be expressed in the typical integral representation [18] [19] [20] [21] [22].

$$
\psi_{n}(C)=\frac{1}{\sqrt{2 \pi}} \int_{-\infty}^{\infty} \mathrm{e}^{i \phi_{n}(s ; C)} \mathrm{d} s,
$$

where

$$
\phi_{n}(s ; C)=s^{n}+\sum_{j=1}^{n-2} C_{j} s^{j},
$$

where $s$ represents the state variable, $C_{j}$ are the $n-2$ control parameters and $\phi_{n}$ is the catastrophe phase polynomial of n-th order that depends on the state variable $s$ and the control parameters $C_{j}$ with the codimension $K=n-2$.

The three members of the hierarchy catastrophe structures that we will examine in this paper are given in Table 1, where we have listed the considered diffraction catastrophes with the corresponding phase polyomials $\phi$ and codimensions $K$. For the sake of comparison we have reported, the Berry's [18] and Stewart's [19] unfolding phase polyomials.

By using the change of the variable $x=\frac{s}{n^{1 / n}}$, one obtains the relationship between the control parameters $C$ and $\Delta$ in the Refs. [18] and [19]. For $K=1$, the phenomenon is called Fold diffraction caustic, we have

$$
C=\frac{a}{3^{1 / 3}}
$$

and the structure function $\psi_{1}(C)$ is proportional to the well-known Airy function

\begin{tabular}{|c|c|c|c|}
\hline $\begin{array}{l}\text { Diffraction } \\
\text { catastrophe }\end{array}$ & $\begin{array}{c}\text { Codimension } \\
K\end{array}$ & Berry's unfolding $\phi(s ; C)[18]$ & $\begin{array}{c}\text { Stewart's } \\
\text { unfolding } \phi(x ; \Delta) \quad[19]\end{array}$ \\
\hline Fold & 1 & $\frac{s^{3}}{3}+C s$ & $x^{3}+a x$ \\
\hline Swallowtail & 3 & $\frac{s^{5}}{5}+C_{3} \frac{s^{3}}{3}+C_{2} \frac{s^{2}}{2}+C_{1} s$ & $x^{5}+a x^{3}+b x^{2}+c x$ \\
\hline Wigwam & 5 & $\frac{s^{7}}{7}+C_{5} \frac{s^{5}}{5}+C_{4} \frac{s^{4}}{4}+C_{3} \frac{s^{3}}{3}+C_{2} s^{2}+C_{1} s$ & $x^{7}+a x^{5}+b x^{4}+c x^{3}+d x^{2}+e x$ \\
\hline
\end{tabular}

Table 1. The elementary diffraction catastrophes of odd codimensions $K \leq 5$ : with Berry and Stewart unfoldings. 


$$
\psi_{1}(C)=\frac{1}{\sqrt{2 \pi}} \int_{-\infty}^{+\infty} \mathrm{d} s \cdot \mathrm{e}^{i\left(\frac{s^{3}}{3}+C S\right)}=\sqrt{2 \pi} A i(C),
$$

where the Airy function is defined as [23],

$$
A i(x)=\frac{1}{\pi} \int_{0}^{+\infty} \mathrm{d} t \cdot \cos \left(\frac{t^{3}}{3}+x t\right) .
$$

For $K=3$, the phenomenon is called Swallowtail diffraction catastrophe [24] [25] [26] [27] [28], so we have

$$
C_{1}=\frac{1}{5^{1 / 5}} c, C_{2}=\frac{2}{5^{2 / 5}} b, C_{3}=\frac{3}{5^{3 / 5}} a,
$$

and the caustic function reads

$$
\psi_{3}\left(C_{1}, C_{2}, C_{3}\right)=\frac{1}{\sqrt{2 \pi}} \int_{-\infty}^{+\infty} \mathrm{d} s \cdot \mathrm{e}^{i\left(\frac{s^{5}}{5}+C_{3} \frac{s^{3}}{3}+C_{2} \frac{s^{2}}{2}+C_{1} s\right)} .
$$

The case $K=5$ corresponds to the so-called Wigwam diffraction catastrophe [24] and we obtain the following identities

$$
C_{1}=\frac{1}{7^{1 / 7}} e, C_{2}=\frac{1}{7^{2 / 7}} d, C_{3}=\frac{3}{7^{3 / 7}} c, C_{4}=\frac{4}{7^{4 / 7}} b \text { and } C_{5}=\frac{5}{7^{5 / 7}} a \text {. }
$$

and

$$
\psi_{5}\left(C_{1}, C_{2}, C_{3}, C_{4}, C_{5}\right)=\frac{1}{\sqrt{2 \pi}} \int_{-\infty}^{+\infty} \mathrm{d} s \cdot \mathrm{e}^{i\left(\frac{s^{7}}{7}+C_{5} \frac{s^{5}}{5}+C_{4} \frac{s^{4}}{4}+C_{3} \frac{s^{3}}{3}+C_{2} \frac{s^{2}}{2}+C_{1} s\right)} .
$$

It is worth noting that, apart from the case $K=1$, the caustic functions $\psi_{n}$ above haven't been expressed, to the best of our knowledge, in terms of well-known mathematical functions. In the remainder of the paper, we will show the relationship between these catastrophe functions and the Airy-Hardy integrals, and propose an optical method by using tailored binary screens, for the creation of the elementary optical catastrophes of odd codimensions $(K=1$, 3 and 5).

\section{Caustic Functions and Their Connection with the Airy-Hardy Integrals}

One can note that for particular values of the control parameters, the phase functions in Equations (6) and (7) are reduced to odd polynomials, and thus the associated catastrophe functions are proportional to the Airy-Hardy cosine integrals [23]. In fact, if one takes $C_{2}=0$ in the case $K=3$, or $C_{2}=C_{4}=0$ for $K=$ 5 , the associated phase polynomials $\phi_{3}$ and $\phi_{5}$ will be odd functions, then it will be simpler to express the caustic functions $(K=1,3$ or 5$)$ as

$$
\begin{gathered}
\psi_{1}(C)=\sqrt{\frac{2}{\pi}} \int_{0}^{+\infty} \mathrm{d} s \cdot \cos \left(\frac{s^{3}}{3}+C s\right), \\
\psi_{3}\left(C_{1}, C_{3}\right)=\frac{2}{\sqrt{2 \pi}} \int_{0}^{+\infty} \mathrm{d} s \cdot \cos \left(\frac{s^{5}}{5}+C_{3} \frac{s^{3}}{3}+C_{1} s\right),
\end{gathered}
$$


and

$$
\psi_{5}\left(C_{1}, C_{3}, C_{5}\right)=\sqrt{\frac{2}{\pi}} \int_{0}^{+\infty} \mathrm{d} s \cdot \cos \left(\frac{s^{7}}{7}+C_{5} \frac{s^{5}}{5}+C_{3} \frac{s^{3}}{3}+C_{1} s\right)
$$

These last integrals belong to the Airy-Hardy integrals class and then they can be expressed in the canonical form [22] [23]

$$
C h_{n}(\alpha)=\int_{0}^{+\infty} \mathrm{d} t \cdot \cos \left[T_{n}(t, \alpha)\right]
$$

where $C h_{n}(\alpha)$ denotes the Airy-Hardy function and

$$
T_{n}(t, \alpha)=t_{2}^{n} F_{1}\left(-\frac{1}{2} n, \frac{1-n}{2} ; 1-n ;-\frac{4 \alpha}{t^{2}}\right) \text {, for } n \geq 2
$$

where ${ }_{2} F_{1}($.$) is the hypergeometric function defined by [29]$

$$
{ }_{2} F_{1}(a, b ; c ; z)=\sum_{n=0}^{\infty} \frac{(a)_{n}(b)_{n}}{(c)_{n}} \frac{z^{n}}{n !},
$$

where (.) is the Pochhammer symbol.

The first odd-order functions $T_{n}(t, \alpha)$ are given by

$$
\left\{\begin{array}{l}
T_{3}(t, \alpha)=t^{3}+3 \alpha t \\
T_{5}(t, \alpha)=t^{5}+4 \alpha t^{3}+5 \alpha^{2} t \\
T_{7}(\alpha)=t^{7}+7 \alpha t^{5}+14 \alpha^{2} t^{3}+7 \alpha^{3} t
\end{array}\right.
$$

It is well-known that the Airy-Hardy functions $C h_{n}($.$) are solutions of the$ following differential equation [22] [23]

$$
\frac{\mathrm{d}^{2} C h_{n}(\alpha)}{\mathrm{d} \alpha^{2}}-n^{2} \alpha^{n-2} C h_{n}(\alpha)=0
$$

Hobbs et al. [23] have expressed these solutions in terms of Bessel functions of fractional orders as

$$
C h_{n}(\alpha)=\frac{\pi|\alpha|^{1 / 2}}{2 n \sin \left(\frac{\pi}{2 n}\right)} \times \begin{cases}I_{-1 / n}\left(2 \alpha^{n / 2}\right)-I_{1 / n}\left(2 \alpha^{n / 2}\right) & \text { for } \alpha>0 \\ J_{-1 / n}\left(2|\alpha|^{n / 2}\right)+J_{1 / n}\left(2|\alpha|^{n / 2}\right) & \text { for } \alpha<0\end{cases}
$$

where $J_{v}$ and $I_{v}$ are the $v$-th order Bessel and the modified Bessel functions of the first kind, respectively.

For $\alpha=0$, the approximate value of $C h_{n}(\alpha)$ reads

$C h_{n}(0) \approx \frac{2}{(2 n+1)^{2 n /(2 n+1)}} \Gamma\left(\frac{1}{2 n+1}\right) \cos \left[\frac{\pi}{2(2 n+1)}\right]$, for $n=1,2$ and 3

By putting $\alpha=\frac{C}{3^{2 / 3}}$ and using the similarity between the Equations and (16a), we obtain

$$
\psi_{1}(\alpha)=\frac{3^{1 / 3}}{\sqrt{2 \pi}} C h_{3}(\alpha) .
$$


The integral expression of Equation (9) can be rewritten

$$
\psi_{3}\left(C_{1}, C_{3}\right)=\frac{2 \cdot 5^{1 / 5}}{\sqrt{2 \pi}} \int_{0}^{+\infty} \mathrm{d} t \cdot \cos \left(t^{5}+\frac{C_{3}}{3} \cdot 5^{3 / 5} t^{3}+C_{1} \cdot 5^{1 / 5} t\right) .
$$

If we take $\frac{C_{3}}{3} 5^{\frac{3}{5}}=4 \alpha$ and $C 5^{\frac{1}{5}}=5 \alpha^{2}$, the last diffraction integral reduces to

$$
\psi_{3}(\alpha)=\frac{2 \cdot 5^{1 / 5}}{\sqrt{2 \pi}} \int_{0}^{+\infty} \mathrm{d} t \cdot \cos \left[T_{5}(t, \alpha)\right]
$$

or equivalently

$$
\psi_{3}(\alpha)=\frac{5^{1 / 5}}{\sqrt{2 \pi}} C h_{5}(\alpha)
$$

The Wigwam catastrophe integral can also be expressed as

$$
\psi_{5}(\alpha)=\frac{2 \cdot 7^{1 / 7}}{\sqrt{2 \pi}} \int_{0}^{+\infty} \mathrm{d} t \cdot \cos \left(t^{7}+\frac{C_{5}}{5} 7^{5 / 7} t^{5}+\frac{C_{3}}{3} 7^{3 / 7} t^{3}+C_{1} 7^{1 / 7} \alpha^{3} t\right) .
$$

By putting $7 \alpha=\frac{7^{5 / 7}}{5} C_{5}, 14 \alpha^{2}=\frac{7^{3 / 7}}{3} C_{3}, 7 \alpha^{3}=7^{1 / 7} C_{1}$, this leads to

$$
\psi_{5}(\alpha)=7^{\frac{1}{7}} \sqrt{\frac{2}{\pi}} C h_{7}(\alpha) .
$$

We inferred from the above procedure that the caustics of odd codimensions $K=1,3$ and 5 are connected to the Hardy-Airy functions of orders $n=3,5$ and 7 , respectively. In the forthcoming section, we will be interested in displaying in optical way these non-conventional functions.

\section{Generation of the Elementary Optical Catastrophes with $K=1,3$ and 5}

Based on the fact that the complex caustic structures are expressed in terms of polynomial phase functions, we adopt an optical technic similar to that employed by Lohmann et al. [2] [3] for implementing Airy, Bessel and Laguerre functions. The proposed setup is a Fourier transformer whose schematic diagram is given in Figure 1: A plane wave front illuminates a binary screen which is transparent only along the curve $y=F(x)$ on the $X Y$ plane. Thus, the transmittance function $T(x, y)$ in the binary screen can be expressed as

$$
T(x, y)=\delta(y-F(x)),
$$

where $F(x)$ is the curve profile and $\delta($.$) is the Dirac function.$

The amplitude distribution of the Fraunhofer diffraction pattern in the back focal plane of the lens $(L)$ is obtained by taking the Fourier transform of Equation (19). This leads to

$$
\hat{T}(\nu, \mu)=\iint T(x, y) \mathrm{e}^{-2 i \pi(\nu x+\mu y)} \mathrm{d} x \mathrm{~d} y .
$$




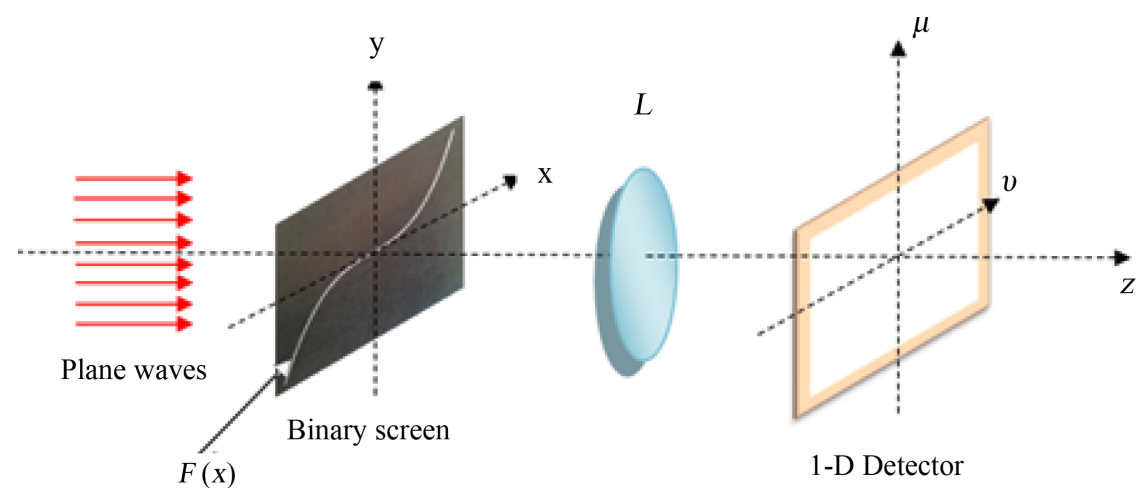

Figure 1. Diagram of the optical setup: at the input plane $X Y$ a binary screen with the transmittance $T(x, y)=\delta(y-F(x))$ illuminated by a plane wave. Detector for output pattern at the back focal plane of the lens $L$.

Substituting from Equation (19) into Equation (20a) and using the integral property of the Dirac function yields

$$
\hat{T}(v, \mu)=\int_{-\infty}^{+\infty} \mathrm{d} x \mathrm{e}^{-2 i \pi(\nu x+\mu F(x))} .
$$

It is worth noting that Equation (20b) is a typical integral representation for many special functions employed is lasers physics, e.g., the Airy, Bessel, Laguerre functions [2] [3]. In particular, if $F(x)$ is odd function, the Equation (20b) can be rewritten as

$$
\hat{T}(v, \mu)=2 \int_{0}^{+\infty} \mathrm{d} x \cdot \cos 2 \pi(v x+\mu F(x)) .
$$

In the following, we propose the transmission profiles that may create oscillating integral patterns encountered in many fields, e.g, in physics, chemistry and biology.

\subsection{The Fold Catastrophe Pattern}

First, let us consider a mask transmission profile of the form $T(x, y)=\delta\left(y-\sigma x^{3}\right)$, where $\sigma$ is an arbitrary constant (Figure 2(a)).

The Fourier transform of this input amplitude can be written as

$$
\hat{T}_{f}(v, \mu)=2(\sigma \mu)^{-1 / 3} C h_{3}\left(\frac{\alpha}{3^{2 / 3}}\right),
$$

with $\alpha=\frac{v}{3}(\sigma \mu)^{-1 / 3}$. The irradiance distribution of the Fraunhofer pattern in the output plane is depicted in Figure 2(b).

By placing a narrow slit in the output plane along the $v$-axis, at $\mu=\eta$ just behind the slit, the output diffracted amplitude reads

$$
\hat{T}_{f}(v, \eta)=\hat{T}_{f}(v, \mu) \delta(\mu-\eta)=2(\sigma \eta)^{-1 / 3} C h_{3}\left(\frac{(\sigma \eta)^{-1 / 3}}{3^{5 / 3}} v\right) .
$$

It follows from this last equation that by displacing the slit to arbitrary values 


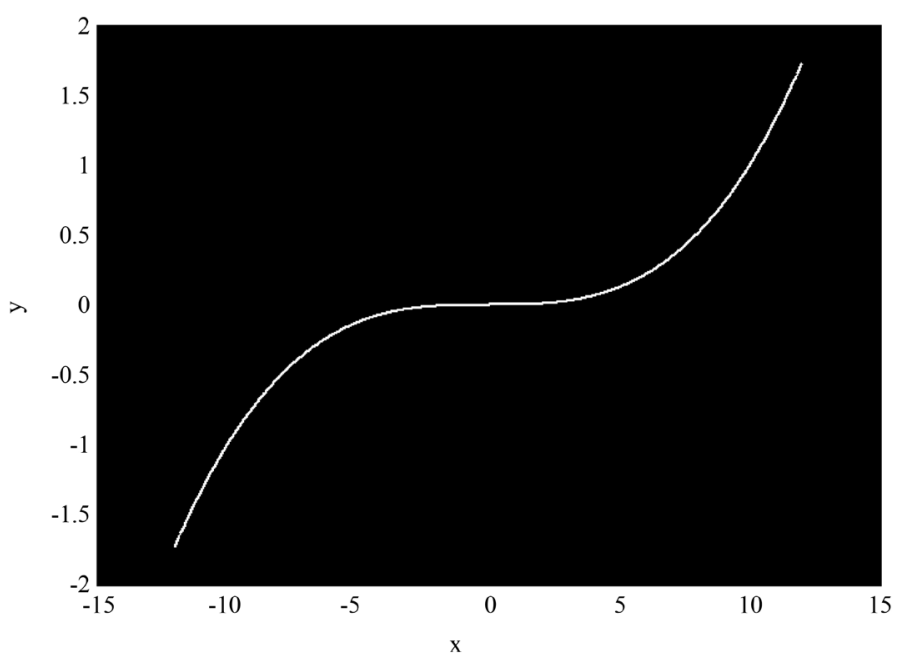

(a)

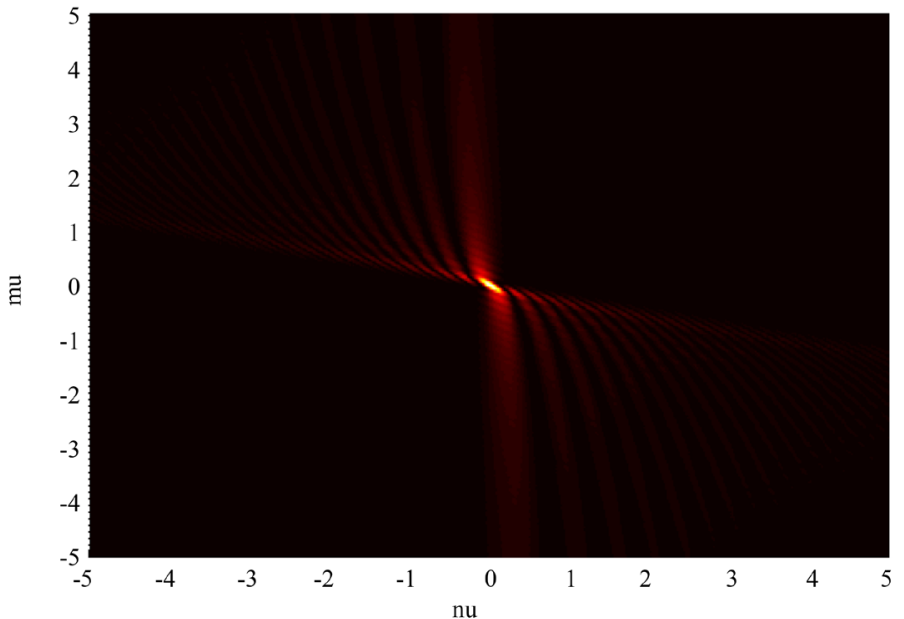

(b)

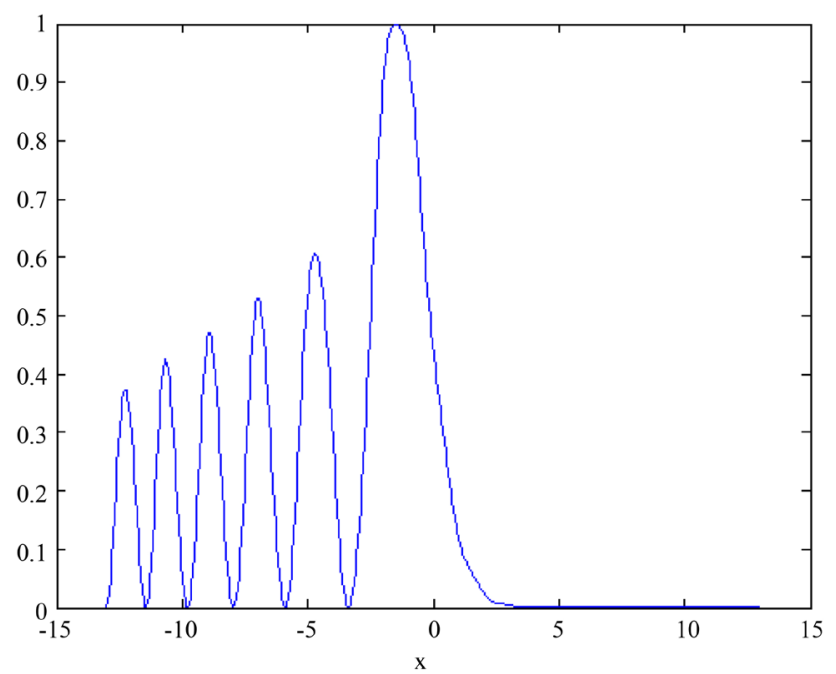

(c)

Figure 2. (a) Input binary pattern with $F(x)=\sigma \cdot x^{3}$, (b) associated output Fraunhofer pattern, and (c) square modulus of Airy-Hardy $\mathrm{Ch}_{3}(x)$ with $v=2$. 
of $\eta$, one can produce the Hardy-Airy function $C h_{3}($.$) along the v$-axis. Figure 2(c) shows the irradiance of $C h_{3}($.$) which is consistent with the$ well-known Airy function, with an arbitrary $\eta$.

\subsection{The Swallowtail Catastrophe Pattern}

Now, by considering the curve function $F_{s}(x)$ of the form (Figure 3(a))

$$
F_{s}(x)=p x^{3}+q x^{5},
$$

where $p$ and $q$ are arbitrary constants, the Fourier transform of the associated input amplitude reads

$$
\hat{T}_{S}(v, \mu)=2 \int_{0}^{+\infty} \mathrm{d} x \cos \left(2 \pi v x+2 \pi \mu p x^{3}+2 \pi \mu q x^{5}\right) .
$$

Making the change of the variable $x=a^{-1 / 5} t$, with $a=2 \pi \mu q$, this leads to

$$
\hat{T}_{S}(v, \mu)=2 a^{-1 / 5} \int_{0}^{+\infty} \mathrm{d} t \cos \left(t^{5}+2 \pi \mu p a^{-3 / 5} t^{3}+2 \pi \mu q a^{-1 / 5} t\right) .
$$

Putting $4 \alpha=2 \pi \mu p \cdot a^{-3 / 5}$ and $5 \alpha^{2}=2 \pi v \cdot a^{-1 / 5}$, and after some algebraic operations, Equation (25) is reduced to

$$
\hat{T}_{s}(v, \mu)=\hat{T}(\alpha)=2 a^{-1 / 5} C h_{5}(\alpha) .
$$

Reciprocally, one can writes

$$
C h_{5}(\alpha)=\frac{1}{2}(2 \pi \mu q)^{1 / 5} \hat{T}_{s}(v, \mu) .
$$

The irradiance distribution of the Fraunhofer pattern of the input amplitude is shown in Figure 3(b). By placing a narrow slit along the $v$-axis at $\mu=\xi$, just behind the slit, the resulted amplitude reads

$$
\hat{T}_{s}(v, \xi)=\hat{T}_{s}(v, \xi) \delta(\mu-\xi)=2(2 \pi \xi q)^{-1 / 5} C h_{5}\left(\frac{5}{4} \frac{v}{\xi p}(2 \pi \xi q)^{2 / 5}\right) .
$$

Hence, one can visualize the profile of the Hary-Airy function $C h_{5}(\alpha)$ along the $v$-axis. Figure $3(\mathrm{c})$ shows the irradiance profile proportional to $C h_{5}(\alpha)$ with $v=2$.

\subsection{The Wigwam Catastrophe Pattern}

By considering the mask transmission function $F_{w}$

$$
F_{W}(x)=p x^{7}+q x^{5}+r x^{3},
$$

where $p, q$ and $r$ are arbitrary constants, its Fourier transform is given by

$$
\hat{T}_{w}(v, \mu)=2 \int_{0}^{+\infty} \mathrm{d} x \cos \left(2 \pi \mu r x^{7}+2 \pi \mu q x^{5}+2 \pi \mu p x^{3}+2 \pi v x\right) .
$$

Now, making the change of variable $x=b^{-1 / 7} t$, with $b=2 \pi \mu r$, and by taking $7 \alpha=\frac{C_{5}}{5} 7^{3 / 7}=2 \pi \mu q b^{-5 / 7}, 14 \alpha^{2}=\frac{C_{5}}{3} 7^{3 / 7}=2 \pi \mu p b^{-3 / 7}$, 


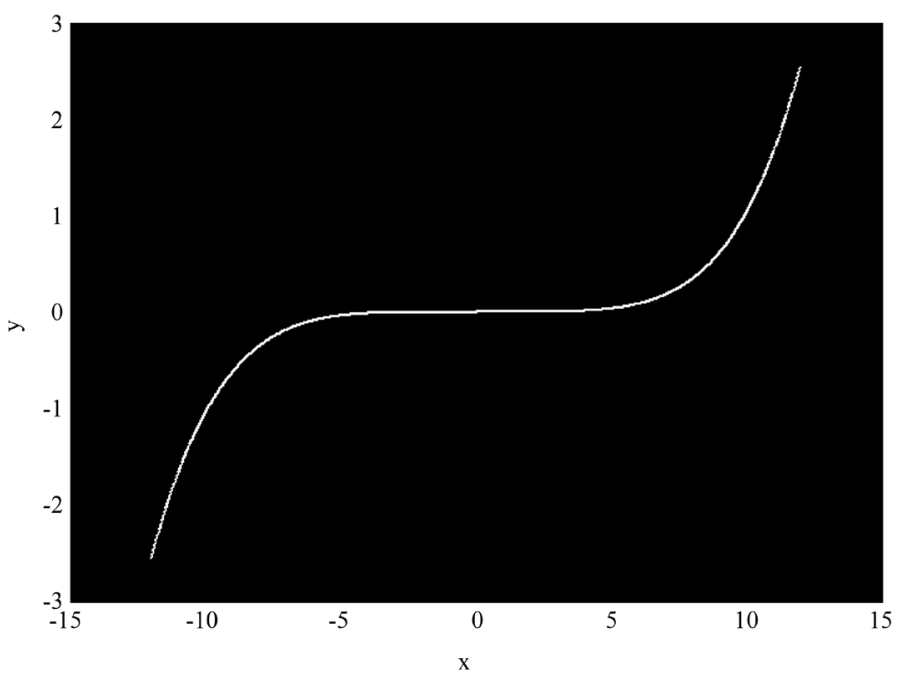

(a)

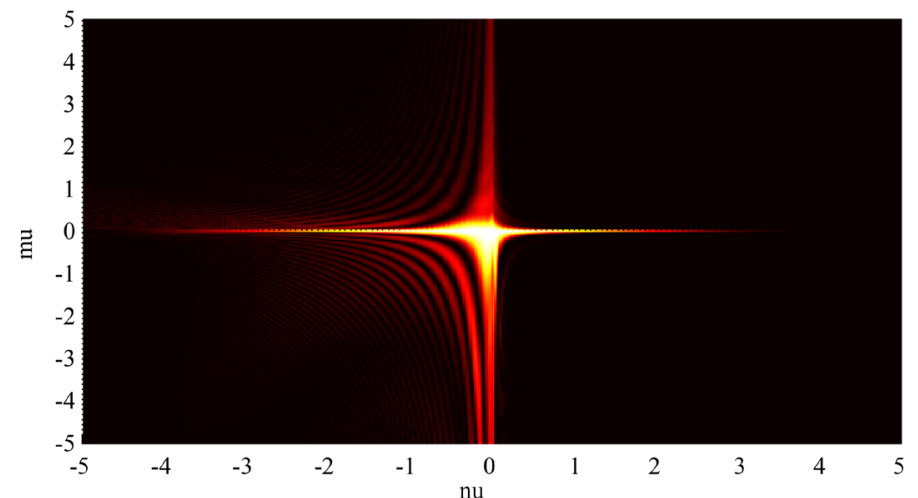

(b)

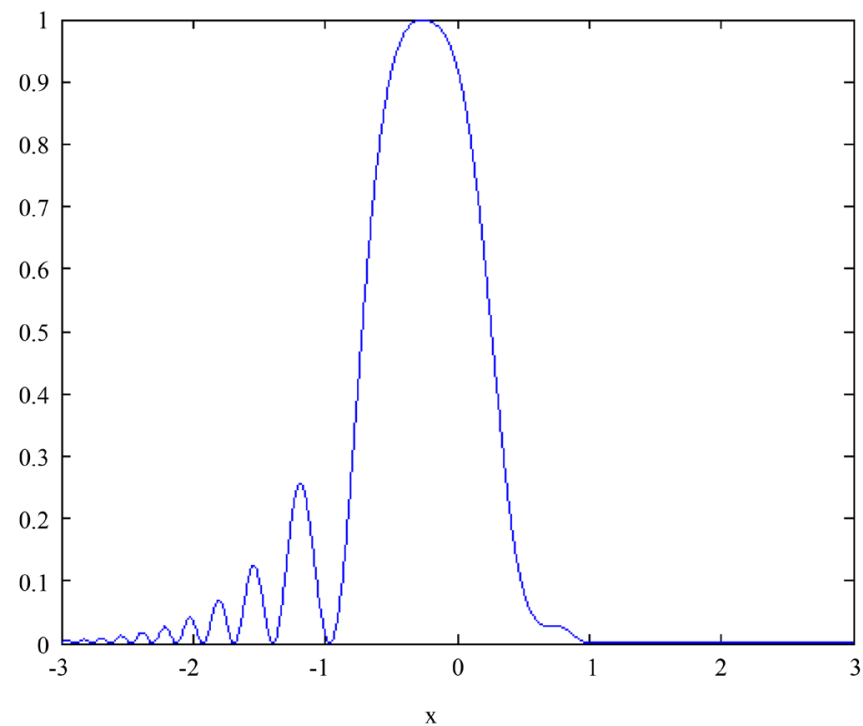

(c)

Figure 3. (a) Input pattern with $F(x)=p \cdot x^{3}+q \cdot x^{5}$, (b) associated output Fraunhofer pattern and (c) square modulus of Airy-Hardy $C h_{5}(x)$ with $v=2$. 
$7 \alpha^{3}=C_{1} 7^{3 / 7}=2 \pi v b^{-1 / 7}$, Equation (30) can be expressed as

$$
\hat{T}_{w}(v, \mu)=2 \cdot b^{-1 / 7} C h_{7}(\alpha),
$$

or equivalently

$$
C h_{7}(\alpha)=\frac{1}{2}(2 \pi \mu q)^{1 / 7} \hat{T}_{s}(v, \mu) .
$$

Following the same procedure as described in Sections (3.1) and (3.2) and using the binary mask of Equation (29), we have displayed the irradiance distribution of the Fraunhofer pattern associated with $\mathrm{Ch}_{7}($.$) in Figure 4(c). It is$

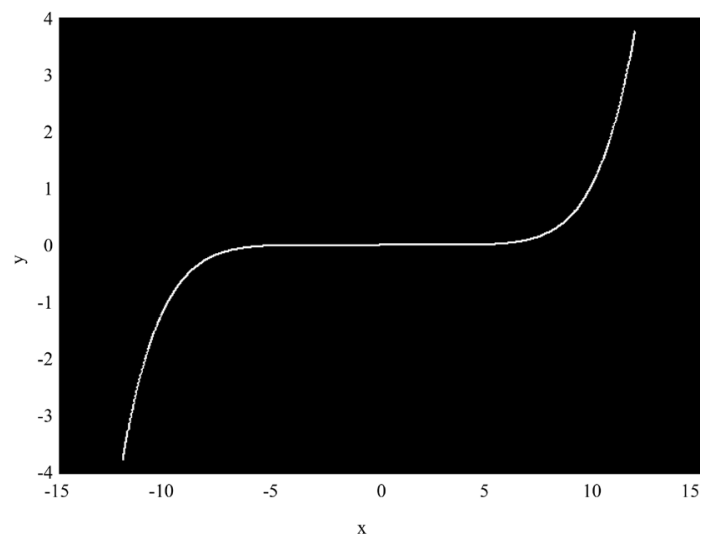

(a)

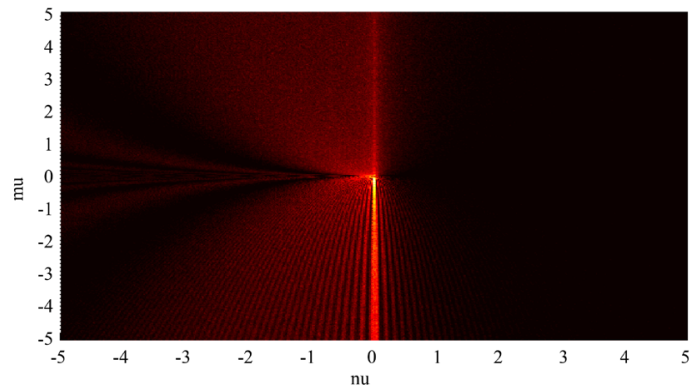

(b)

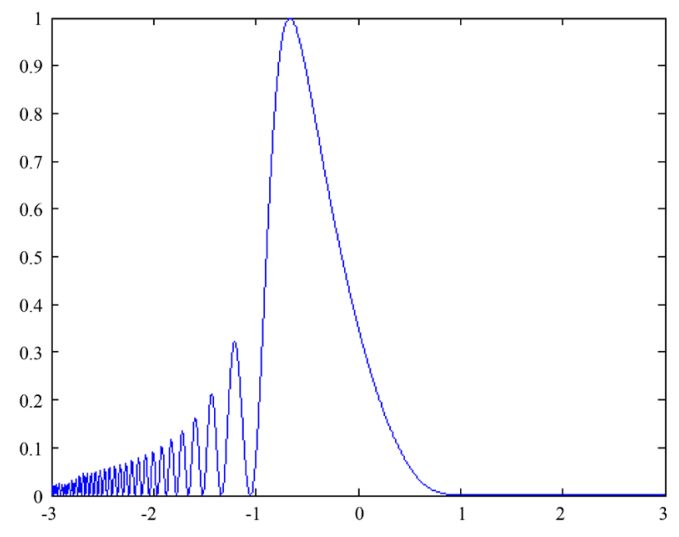

(c)

Figure 4. (a) Input pattern with $F(x)=p \cdot x^{7}+q \cdot x^{5}+r x^{3}$, (b) associted output Fraunhofer pattern and (c) square modulus of Airy-Hardy $\mathrm{Ch}_{7}(x)$ with $v=2$. 
worth noting that the patterns of Figure 2(c), Figure 3(c) and Figure 4(c) can be controlled by adjusting the parameters $\sigma, p, q, r$, e.g., a negative value of $\sigma$ will give the symmerical profile of Figure 2(c).

The parameter $\sigma$ can be regarded as a scaling length for controlling the Fraunhofer pattern structure of Figure 2(b), and in the same way, different mapping caustics for Swallowtail and Wigwam optical catastrophes (Figure 3(c) and Figure $4(\mathrm{c})$ ) can be performed, globally, by varying the scaling parameters $p, q$ and $r$ of the binary amplitude transmittance in the input plane This procedure may permit application opportunities in micromachining on tailored curves and light guiding paths.

\section{Conclusion}

In summary, based on the fact that the integral diffraction catastrophes can be reduced in the case of odd polynomials phase functions into Airy-Hardy cosine integrals, we obtained the closed-form expressions for the related caustic functions. We showed that by the use of an optical Fourier Transformer device with tailored binary screens one can obtain the displaying of caustic beams such as the Fold, the Swallowtail and the Wigwam catastrophes. Furthermore, it is shown that the mapping caustics for Swallowtail and Wigwam caustics can be controlled by the scaling parameters $p, q$ and $r$ of the binary mask transmittance. The result of this work can be useful in shaping lasers with catastrophe amplitude function and may have application opportunities in micromachining and light guiding paths. This study can be extended to higher oscillatory integral functions.

\section{Conflicts of Interest}

The authors declare no conflicts of interest regarding the publication of this paper.

\section{References}

[1] Goodman, J.W., Kellmannad, P. and Hansen, E.W. (1977) Linear Space-Variant Optical Processing of 1-D Signals. Applied Optics, 16, 733-738. https://doi.org/10.1364/AO.16.000733

[2] Lohmann, A.W., O-Castaneda, J. and Heredia, A.S. (1994) Bessel Functions and Laguerre Polynomials: Parallel Display and Processing. Optics Letters, 19, 55-57. https://doi.org/10.1364/OL.19.000055

[3] Lohmann, A.W., O-Castaneda, J. and Ibarra, J.G. (1994) Airy Function and Laguerre Polynomials: Optical Display and Processing. Optics Communications, 109, 361-367. https://doi.org/10.1016/0030-4018(94)90482-0

[4] Berry, M.V. and Balazs, N.L. (1979) Nonspreading Wave Packets. American Journal of Physics, 47, 264-267. https://doi.org/10.1119/1.11855

[5] Siviliglou, G.A., Broky, J., Dogariu, A. and Chritodoulides, D.N. (2007) Observation of Accelerating Airy Beams. Physical Review Letters, 99, Article ID: 213901. https://doi.org/10.1103/PhysRevLett.99.213901 
[6] Siviliglou, G.A. and Chritodoulides, D.N. (2007) Accelerating Finite Energy Airy Beams. Optics Letters, 32, 979-981. https://doi.org/10.1364/OL.32.000979

[7] Ez-Zariy, L., Nebdi, H., Boustimi, M. and Belafhal, A. (2014) Transformation of a Two-Dimensional Finite Energy Airy Beam by an ABCD Optical System with a Rectangular Annular Aperture. Physical and Chemical News, 73, 39-49.

[8] Ez-Zariy, L., Hennani, S., Nebdi, H. and Belafhal, A. (2014) Propagation Characteristics of Airy-Gaussian Beams Passing through a Misaligned Optical System with Finite Aperture. Optics and Photonics Journal, 4, 325-336. https://doi.org/10.4236/opj.2014.411033

[9] Ebrahim, A.A.A., Ez-Zariy, L. and Belafhal, A. (2015) Propagation of Finite Airy-Hermite Hollow Gaussian Beams through a Paraxial ABCD Optical System. International Journal of Advancement in Earth and Environmental Sciences, 3, 11-20.

[10] Ez-Zariy, L., Hricha, Z. and Belafhal, A. (2015) Novel Finite Airy Beams Generated from Gaussian Array Beams Illuminating an Optical Airy Transform System. Progress in Electromagnetics, 49, 41-50. https://doi.org/10.2528/PIERM16041909

[11] Ez-Zariy, L., Boufalah, F., Dalil-Essakali, L. and Belafhal, A. (2018) Conversion of the Hyperbolic-Cosine Gaussian Beam to a Novel Finite Airy-Related Beam Using an Optical Airy Transform System. Optik, 171, 501-506.

https://doi.org/10.1016/j.ijleo.2018.06.091

[12] Ouahid, L., Dalil-Essakali, L. and Belafhal, A. (2018) Effect of Light Absorption and Temperature on Self-Focusing of Finite Airy-Gaussian Beams in a Plasma with Relativistic and Ponderomotive Regime. Optical and Quantum Electronics, 50, 216. https://doi.org/10.1007/s11082-018-1483-3

[13] Ouahid, L., Dalil-Essakali, L. and Belafhal, A. (2018) Relativistic Self-Focusing of Finite Airy-Gaussian Beams in Collisionless Plasma Using the Wentzel-KramersBrillouin Approximation. Optik, 154, 58-66. https://doi.org/10.1016/j.ijleo.2017.10.009

[14] Yaalou, M., Hricha, Z., El Halba, E.M. and Belafhal, A. (2019) Production of Airy-Related Beams by an Airy Transform Optical System. Optical and Quantum Electronics, 51, 308. https://doi.org/10.1007/s11082-019-2023-5

[15] Yaalou, M., Hricha, Z. and Belafhal, A. (2019) Propagation Properties of Finite Cosh-Airy Beams through an Airy Transform Optical System. Optical and Quantum Electronics, 51, 356. https://doi.org/10.1007/s11082-019-2075-6

[16] Ring, J.D., Lindberg, J., Mourka, A., Mazilu, M., Dholakia, K. and Denis, M.R. (2012) Autofocusing and Self-Healing of Pearcey Beams. Optics Express, 20, 18955-18966. https://doi.org/10.1364/OE.20.018955

[17] Boufalah, F., Dalil-Essakali, L., Nebdi, H. and Belafhal, A. (2016) Effect of Turbulent Atmosphere on the On-Axis Average Intensity of Pearcey-Gaussian Beam. Chinese Physics B, 25, Article ID: 064208. https://doi.org/10.1088/1674-1056/25/6/064208

[18] Berry, M.V. and Upsill, C. (1980) Catastrophe Optics: Morphologies of Caustics and Their Diffraction Patterns. Progress in Optics, 18, 257-346. https://doi.org/10.1016/S0079-6638(08)70215-4

[19] Stewart, I. (1981) Applications of Catastrophe Theory to Physical Sciences. Physica D: Nonlinear Phenomena, 2, 245-305. https://doi.org/10.1016/0167-2789(81)90012-9

[20] Kravtsov, Y.A. and Orlov, Yu.I. (1983) Caustics, Catastrophes, and Wave Fields. Uspekhi Fizicheskikh Nauk, 141, 591-627.

https://doi.org/10.3367/UFNr.0141.198312b.0591 
[21] Nye, J.F. (1999) Natural Focusing and Five Structures of Light: Caustics and Wave Dislocation. IOP Publishing, Bristol.

[22] Hobbs, C.A., Connor, J.N.L and Kirk, N.P. (2007) Theory and Numerical Evaluation of Oddoids and Evenoids: Oscillatory Cuspoid Integrals with Odd and Even Polynomial Phase Functions. Journal of Computational and Applied Mathematics, 207, 192-213. https://doi.org/10.1016/j.cam.2006.10.079

[23] Watson, G.N. (1944) A Treatise of the Theory of Bessel Functions. Cambridge University Press, Cambridge.

[24] Zannotti, A., Diebel, F., Boguslawski, M. and Denz, C. (2017) Optical Catastrophes of the Swallowtail and Butterfly Beams. New Journal of Physics, 19, Article ID: 053004. https://doi.org/10.1088/1367-2630/aa6ecd

[25] Zannotti, A., Diebel, F. and Denz, C. (2017) Dynamics of Optical Swallowtail Catastrophe. Optica, 4, 1157-1162. https://doi.org/10.1364/OPTICA.4.001157

[26] Zannoti, A., Ruschenbaum, M. and Denis, C. (2017) Dynamics of Optical Swallowtail Catastrophe. Journal of Optics, 19, Article ID: 94001.

[27] Cheng, K., Lu, G. and Zhong, X. (2017) The Poynting Vector and Angular Momentum Density of the Swallowtail-Gauss Beams. Optics Communications, 396, 58-65. https://doi.org/10.1016/j.optcom.2017.03.038

[28] Cheng, K., Lu, G., Zhou, Y., Yao, N. and Zhong, X. (2018) The Poynting Vector and Angular Momentum Density of the Autofocusing Butterfly-Gauss Beams. Optics and Laser Technology, 105, 23-34. https://doi.org/10.1016/j.optlastec.2018.02.029

[29] Abramowitz, M. and Stegun, I.A. (1964) Handbook of Mathematical Functions. Nath. Bureau of Standards, Washington DC. 